\title{
Epidemiología de la enfermedad de Chagas: alta mortalidad y tasa de incidencia, Región de Coquimbo
}

\section{Epidemiology of Chagas disease: high mortality and incidence rate, Coquimbo Region}

\author{
Paola Salas $R^{1,2}$ \\ 'Núcleo de Investigación y Transferencia Tecnológica Área Salud, Facultad de Ciencias, Universidad de La Serena. La Serena, Chile. \\ ${ }^{2}$ Departamento de Enfermería, Facultad de Ciencias, Universidad de La Serena.
}

Financiamiento: Facultad de Ciencias Universidad de La Serena.

Recibido: 2 de febrero de 2020 / Aceptado: 13 de julio de 2020

\section{Resumen}

Introducción: La enfermedad de Chagas en Chile ha aumentado en pesquisa, especialmente en mujeres gestantes y donantes en general, pero sigue desatendida en Latinoamérica. La epidemiología ambiental del vector señala a la Región de Coquimbo de mayor riesgo. Objetivo: Analizar la serie temporal de mortalidad y tasa de incidencia por enfermedad de Chagas a nivel nacional, regional, comunal y según edad. Método: Estudio de base poblacional, serie de tiempo de mortalidad (1997 al 2017) y tasa de incidencia (2011 al 2017), país, región y 15 comunas. Uso de tasas brutas y ajustadas, calculando $\chi^{2}$, T test, ANOVA, valor $\mathrm{p}<0,05$. Resultados: Mortalidad; El 49,37\% (668) de las muertes nacionales (1.353) tenían residencia en la Región de Coquimbo, mayoritariamente hombres y mayores de 60 años con complicaciones cardiacas y digestivas, residentes en Salamanca, Combarbalá e Illapel. Incidencia; De 6.173 casos acumulados, $6 \%$ son menores de 20 años, mayor en mujeres $(61,4 \%)$ con edad promedio 50 años versus 56 años hombres ( $\mathrm{T}$ test $=29,19$ valor $\mathrm{p}=0,000)$, residentes de; Ovalle, Andacollo, Monte Patria e Illapel. Conclusiones: La mitad de las muertes por enfermedad de Chagas residen en la Región de Coquimbo, los casos nuevos son 6\% en personas bajo 20 años de edad.

Palabras clave: enfermedad de Chagas; mortalidad; incidencia; Coquimbo; Chile.

\section{Introducción}

$\mathrm{E}$ n Latinoamérica se estima que entre seis y once millones de personas están afectadas por la enfermedad de Chagas. Esta parasitosis es considerada una patología desatendida pues, menos de $10 \%$ de las personas en las Américas han sido diagnosticadas

\begin{abstract}
Background: Chagas disease in Chile has increased in research, especially in pregnant women and donors in general, but remains neglected in Latin America. The environmental epidemiology of the vector points out the Coquimbo region is a greatest risk area. Aim: To analyze the time series of mortality and incidence rate Chagas disease national; regional and communal according to age. Method: Population-based study, time series of mortality (1997 to 2017) and incidence rate (2011 to 2017), country, region and 15 communes. Use of gross and adjusted rates, calculating ch2, T test, ANOVA, $\mathrm{p}$ value $<0.05$. Results: Mortality; $49.37 \%$ (668) of the National deaths (1353) were Coquimbo region's residents, mainly male patients and over 60 years old people with cardiac and digestive complications, residents from Salamanca, Combarbalá and Illapel. Incidence: Of 6.173 accumulated cases, $6 \%$ are under 20 years old, higher incidence of women $(61.4 \%)$ with an average age of 50 years old versus 56 years old men ( T test $=29.19 \mathrm{p}$-value $=0.000)$, residents from Ovalle, Andacollo, Monte Patria and Illapel. Conclusions: Half of Chagas' disease deaths reside in the Coquimbo region, new cases, under 20 years old, are $6 \%$.

Keywords: Chagas disease; mortality; incidence rate; Coquimbo; Chile.
\end{abstract}

$\mathrm{y}$, si bien los medicamentos para su tratamiento se descubrieron hace medio siglo, han sido proporcionado a sólo $1 \%$ de las personas que padecen la enfermedad. A esto se suma, que los determinantes de la salud como la pobreza y las condiciones de la vivienda en las áreas endémicas favorecen la transmisión vectorial ${ }^{1}$.

En Chile, la última encuesta nacional de salud (ENS 2016-2017) 
- Describir la epidemiología de la enfermedad de Chagas en las personas, a través de analizar la serie temporal de las tasas de mortalidad y la tasa de incidencia (estimada por los casos confirmados por el ISP) en la Región de Coquimbo y sus 15 comunas y,

- En el caso de la mortalidad, incluir además al país. la Región de Coquimbo, la prevalencia alcanzó a $2,8 \%$ (cifra mayor a la ENS 2009-2010 con 1,8\%), lo que podría deberse a un mayor diagnóstico en el país y en la región, especialmente en mujeres gestantes ${ }^{2,3}$.

La enfermedad de Chagas se ha enfrentado en dos ámbitos complementarios en nuestro país; por una parte, con el programa de control vectorial y por otra, con el programa de prevención y control de la enfermedad en las personas. El primero incluye el logro de la interrupción de la transmisión vectorial, certificada en 1999 y con re-certificación en el año 2016 por la Organización Panamericana de la Salud (OPS) ${ }^{3}$. En las personas se implementó la notificación obligatoria (ENO) de los casos confirmados como positivos por el Instituto de Salud Pública de Chile (ISP) desde 1986 y, diez años después, se hizo obligatorio el análisis de Trypanosoma cruzi en la sangre de donantes de las zonas endémica (1996). En el año 2014 comienza la búsqueda activa en mujeres embarazadas (factor predominante de transmisión de la madres al hijo) y en el 2017 comienza lentamente al acceso al tratamiento tripanomicida en algunos grupos que, según la norma actual corresponde a: personas en fase aguda, hospederos inmunodeprimidos, pacientes bajo 18 años de edad, recién nacidos con enfermedad de Chagas congénito, mujeres en edad fértil hasta 45 años, pacientes jóvenes y adultos hasta los 60 años con serología positiva, en fase crónica indeterminada. En todos estos grupos, el objetivo principal es disminuir la carga parasitaria ${ }^{3-5}$. El evento final epidemiológico de esta enfermedad es la aparición de manifestaciones cardíacas y gastrointestinales que conducen a la muerte.

El último informe epidemiológico publicado en Chile sobre la enfermedad de Chagas señala que la tasa de casos notificados (según el formulario de enfermedad de notificación obligatoria-ENO) ha aumentado en $10 \%$ entre los años 2016 y 2017 en el país (tasas 7,53 versus 8,20 casos por 100 mil habitantes, respectivamente), en especial en los mayores de 60 años. Las tasas más altas se observaron en la Región de Coquimbo (IV Región) la que presenta una tasa de 52,12 por 100 mil habitantes el año 2017, con un aumento de $43,14 \%$ comparado con el año $2016^{6}$.

Se agrega que recientes investigaciones ambientales sitúan a Coquimbo como una de las zonas de mayor riesgo para la presencia de $T$. infestans, asociado a temperatura máxima, y la precipitación durante el mes más seco, con mayor presencia del vector en el Norte de Chile (Región de Coquimbo) ${ }^{6-9}$.

Con base a todos los antecedentes antes descritos, se propuso en esta investigación como objetivos:

\section{Material y Métodos}

\section{Diseño}

Estudio epidemiológico de tendencias (serie de tiempo) de las tasas de mortalidad e incidencia de la enfermedad de Chagas. Las unidades de estudio fueron: el país, la Región de Coquimbo, y las 15 comunas de la región, en todos los cuales se realizó un análisis de tendencias y distribución espacial de las tasas de mortalidad bruta y tasas ajustadas.

\section{Datos}

Los análisis de la mortalidad por enfermedad de Chagas se realizaron con la serie de tiempo de casos y tasas de mortalidad bruta y ajustada, con acceso a las bases de datos del Departamento de Estadísticas de Salud de Chile (DEIS-Minsal), que incluyó el total de variables sociodemográficas disponibles en los certificados de defunción del período 1997 al 2017. Se determinó, además, la variabilidad y porcentaje del total país comparado con la Región de Coquimbo. Se agregó para la estimación de la incidencia, el cálculo de tasas proveniente de la base de datos enviada como casos confirmados con enfermedad de Chagas por parte del ISP, y que fueron facilitados por esta institución a la Universidad de La Serena por Ley de Transparencia correspondiente al período 2011 a 2017, en nóminas individuales anónimas con los casos confirmados del sistema público como privado de la Región de sólo hasta el año 2014 en la página del DEIS-MINSAL; por eso, se pudo comparar el porcentaje de notificados sólo en tres años (2011 al 2014).

\section{Datos de estadísticas vitales}

Las estadísticas de mortalidad fueron extraídas de los certificados de defunción provenientes de personas fallecidas por enfermedad de Chagas, código 086 de la clasificación internacional de enfermedades (CIE-9) y B57 (de la CIE 10). Las bases de datos fueron proporcionadas por el DEIS-MINSAL, y analizados como datos secundarios anónimos ${ }^{10}$.

En la construcción de las tasas se utilizó como denominador las proyecciones anuales de la población-país, Región de Coquimbo y comunas, entregada por el Instituto Nacional de Estadísticas (INE) de Chile ${ }^{11}$.

\section{Análisis estadístico}

En las características socio-demográficas de los casos que han fallecido por enfermedad de Chagas y los que han Coquimbo. Las bases de dato ENO estaban disponibles 
sido diagnosticados y confirmados por el ISP, se estimó diferencias en los promedios de variables continuas como la edad y los años de educación con T test, y cálculo de $\chi^{2}$ en las variables categóricas. Se consideraron diferencias significativas estadísticamente valores de $p<0,05$.

La tasa bruta de mortalidad fue calculada con el número de muertes por la enfermedad de Chagas, dividido por el total de la población de cada año, multiplicado por 100.000. En la tasa de incidencia correspondió a los nuevos casos confirmados por el ISP y enviados en planillas individuales para cada año. Para una mayor representatividad y estabilidad de los datos comunales se decidió, además, calcular tasas acumuladas brutas con el total de casos diagnosticados en el período (20 años en el caso de mortalidad y 7 años en el caso de las tasas de incidencia) y como denominador la población de la mitad del período (estimación del promedio) a junio del año 2006 en mortalidad y 2014 en incidencia. Se comparó la mortalidad del período completo (1997-2017) y el período similar a incidencia (2011-2017) sin mayores variaciones entre los lugares de las magnitudes alcanzadas por comuna; por tal motivo, se decidió continuar con el análisis de tasas de mortalidad del período completo. Para intentar controlar la distorsión que se produce al comparar poblaciones con distinta estructura de edad, se realizó una estandarización de las tasas acumuladas brutas homogenizando las diferentes poblaciones y haciéndolas comparables entre sí, realizando un ajuste directo, tomando como referencia la población nacional de los años de la mitad de los períodos como ya fue mencionado, por grupos de edad, multiplicado por un amplificador de 1.000 habitantes para las tasas de incidencia y de 100.000 habitantes en el caso de la mortalidad, para su mayor comprensión. Se estimó los casos esperados y se procedió a calcular las tasas ajustadas de esta manera, por edad y población estándar del país; posteriormente a este procedimiento se construyó los mapas.

Los mapas fueron coropléticos para graficar la distribución espacial de las 15 comunas de la Región de Coquimbo, utilizando un sistema de información geográfico con tasas acumuladas en ambos períodos de interés, como ya fueron descritas. Los puntos de corte para los intervalos, de acuerdo con la distribución de las tasas ajustada en ambos indicadores, fueron proporcional a seis rangos; estos rangos fueron creados para intentar dirigir la atención en los extremos de la distribución. Las comunas ubicadas en la primera categoría representan las comunas con menores tasas y, en el otro extremo, comunas con riesgos mayores. Para la representación de las categorías en el mapa, se eligieron seis colores en una misma escala de tono azul para tasas de incidencia y rojo para tasas de mortalidad, donde los colores intensos indican alto riesgo, así hasta los colores claro que indican bajo riesgo.

\section{Resultados}

\section{Mortalidad por enfermedad de Chagas en Chile y Región de Coquimbo}

Las características socio-demográficas que los certificados de defunción entregaron de los casos fallecidos entre 1997 y 2017 por causa de la enfermedad de Chagas, señalan que las defunciones ocurrieron principalmente en hombres (casi 10\% más), tanto en el país como en la Región de Coquimbo ( $\mathrm{T}$ test $=5,851$, valor $\mathrm{p}=0,016)$. La edad promedio de muerte de hombres fue 75 años versus 78 años en mujeres, con diferencias significativas. La procedencia de los fallecidos fue principalmente urbana; la diferencia estaba en que en la Región de Coquimbo la razón urbano:rural es de 1,5, y en el país de 5,6, es decir en Coquimbo fallece una mayor proporción de personas del sector rural. La Región de Coquimbo presentó mayor porcentaje de analfabetos (as) y menor educación, secundaria, media y universitaria. El último curso aprobado fue un año menos en los fallecidos de la Región de Coquimbo que el promedio del resto del país. Todas estas diferencias fueron estadísticamente significativas (Tabla 1).

El total de casos fallecidos por enfermedad de Chagas en el período analizado entre 1997 y 2017 correspondió a 1.353 casos en todo el país, de los cuales 668 casos $(49,37 \%)$ presentaron residencia en la Región de Coquimbo. La tendencia en la tasa de mortalidad en esta región ha sido siempre superior al país durante todo el período analizado; por ejemplo, en el año 2017 la tasa de mortalidad bruta en la Región de Coquimbo fue de 4,8 por 100.000 habitantes versus 0,4 por 100.000 habitantes en el resto del país (Figura 1).

En términos de tendencias, se mantiene una variabilidad oscilante en los casos de muerte, siempre con tasas mayores en la Región de Coquimbo que el resto del país, alcanzando un promedio anual de $51,7 \%$ entre los años 1997 y el año 2017 y un rango que va desde $40,4 \%$ en el año 2008 a 63,2\% en el año 2009. A pesar de estas oscilaciones, la proporción de fallecidos cuya residencia es la Región de Coquimbo es considerablemente alta respecto al total de casos cada año (Figura 2).

Respecto a la causa de mortalidad específica de la enfermedad de Chagas, según diagnóstico primario de defunción, el grupo de "Enfermedad de Chagas crónica que altera el corazón” (B57.2) presenta un mayor número de casos cada año respecto a los demás diagnósticos durante todo el período, y tiene una tendencia oscilante al ascenso. Le siguen, en segundo lugar de magnitud, los diagnósticos de muerte por "Enfermedad de Chagas crónica que afecta el aparato digestivo" (B57.3), y en tercer lugar la que afecta a "Otros órganos" (B57.5), (Figura 3).

La mortalidad por la enfermedad de Chagas aumenta a medida que aumenta la edad. Las personas que fallecen a menor edad son también aquellas del grupo de la enfer- 


\begin{tabular}{|c|c|c|c|c|c|c|}
\hline \multirow[t]{2}{*}{ Variables } & \multirow[t]{2}{*}{ Categorías } & \multicolumn{2}{|c|}{ Distribución geográfica } & \multirow[b]{2}{*}{ Total, país } & \multirow[b]{2}{*}{$T$ test $(t) / \chi^{2}$} & \multirow[b]{2}{*}{$\begin{array}{l}\text { Valor p/ } \\
\text { Signif. }\end{array}$} \\
\hline & & Región Coquimbo & $\begin{array}{l}\text { Total, otras } \\
\text { regiones de Chile }\end{array}$ & & & \\
\hline Sexo & Hombre & $55,9 \%(361)$ & $62,4 \%(439)$ & $59,3 \%(800)$ & $\mathrm{T}$ test $=5,8$ & $0,016^{*}$ \\
\hline (Porcentaje y $\mathrm{N}^{\circ}$ ) & Mujer & $44,1 \%(285)$ & $37,6 \%(265)$ & $40,7 \%(550)$ & & \\
\hline Procedencia & Urbanas & $60,2 \%(389)$ & $84,9 \%(598)$ & $73,1 \%(987)$ & $\mathrm{T}$ test $=104,7$ & $0,000 *$ \\
\hline (Porcentaje y $\mathrm{N}^{\circ}$ ) & Rurales & $39,8 \%(257)$ & $15,1 \%(106)$ & $26,9 \%(362)$ & & \\
\hline \multirow{3}{*}{$\begin{array}{l}\text { Actividad } \\
\text { (Porcentaje y } N^{\circ} \text { ) }\end{array}$} & Inactivo (a) & $92,1 \%(595)$ & $90,3 \%(636)$ & $91,2 \%(1231)$ & $\chi^{2}=1,3$ & 0,499 \\
\hline & Activo (a) & $7,6 \%(49)$ & $9,4 \%(66)$ & $8,5 \%(115)$ & & \\
\hline & Cesante & $0,3 \%(2)$ & $0,3 \%(2)$ & $0,3 \%(4)$ & & \\
\hline \multirow{5}{*}{$\begin{array}{l}\text { Estado civil } \\
\text { (Porcentaje y } \mathrm{N}^{\circ} \text { ) }\end{array}$} & Soltero (a) & $36,7 \%(237)$ & $30,7 \%(216)$ & $33,6 \%(453)$ & $\chi^{2}=7,6$ & 0,107 \\
\hline & Casado (a) & $52,0 \%(336)$ & $59,2 \%(417)$ & $55,8 \%(753)$ & & \\
\hline & Viudo (a) & $11 \%(71)$ & $9,7 \%(68)$ & $10,3 \%(139)$ & & \\
\hline & Divorciado & $0,2 \%(1)$ & $0,3 \%(2)$ & $0,2 \%(3)$ & & \\
\hline & Conviviente & $0,2 \%(1)$ & $0,1 \%(1)$ & $0,1 \%(2)$ & & \\
\hline \multirow{5}{*}{$\begin{array}{l}\text { Educación } \\
\text { (Porcentaje y Nº) }\end{array}$} & Analfabeto (a) & $28,2 \%(182)$ & $12,4 \%(87)$ & $19,9 \%(269)$ & $\chi^{2}=66,4$ & $0,000^{*}$ \\
\hline & Básica o Primaria & $59,6 \%(385)$ & $64,8 \%(456)$ & $62,3 \%(841)$ & & \\
\hline & Secundaria & $8,8 \%(57)$ & $14,8 \%(104)$ & $11,9 \%(161)$ & & \\
\hline & Media & $2,6 \%(17)$ & $6,3 \%(44)$ & $4,5 \%(61)$ & & \\
\hline & Superior & $0.8 \%(5)$ & $1,8 \%(13)$ & $1,3 \%(18)$ & & \\
\hline \multirow{3}{*}{$\begin{array}{l}\text { Lugar defunción } \\
\text { (Porcentaje y } \mathrm{N}^{\circ} \text { ) }\end{array}$} & Hospital & $52,6 \%(340)$ & $62,9 \%(443)$ & $58,0 \%(783)$ & $\chi^{2}=14,6$ & $0,001 *$ \\
\hline & Casa & $44,1 \%(285)$ & $34,4 \%(242)$ & $39,0 \%(527)$ & & \\
\hline & Otro & $3,3 \%(21)$ & $2,7 \%(19)$ & $3 \%(40)$ & & \\
\hline Edad (Promedio y DE)** & Edad defunción & $75,26( \pm 12,1)$ & $72,59( \pm 12,1)$ & $73,87( \pm 12,2)$ & $T$ test $=16,3$ & $0,000 *$ \\
\hline Curso aprobado (Promedio y DE)** & Último curso aprobado & $2,99( \pm 2,33)$ & $4,06( \pm 2,17)$ & $3,55( \pm 2,3)$ & T test $=77,04$ & $0,000^{*}$ \\
\hline
\end{tabular}

medad de Chagas crónica que altera el corazón (B57.2) y presenta además una tendencia al ascenso marcada luego de los 45 años, también a los 65 años y los 75 años. En cambio, la mortalidad por causas digestivas se presenta a mayores edades, principalmente luego de los 65 años (Figura 4).

\section{Tasa incidencia de enfermedad de Chagas en la Región de Coquimbo}

La tasa bruta de incidencia por enfermedad de Chagas acumulada entre 2011 y 2017 fue de 8,1 por 1.000 habitantes $(6.173 / 759.228$ habitantes de la región * 1.000), como corresponde a los casos nuevos que se confirman por el ISP cada año, con tendencia anual oscilante y en promedio de 882 casos al año, sumados el sistema público y el privado de la región. Las oscilaciones fluctúan con una variabilidad de hasta 1.077 casos entre los años de análisis (por ejemplo, entre los años 2011 y 2013), descienden en el año 2013 y vuelven a subir en forma paulatina hasta el año 2017, último año completo enviado por el ISP. Suman para todo el período 6.173 casos confirmados del sistema público como privado; de ellos, sólo $2.946(47,7 \%)$ tenían registros de la variable edad (Figura 5).

Respecto a la notificación ENO, la detección de casos 


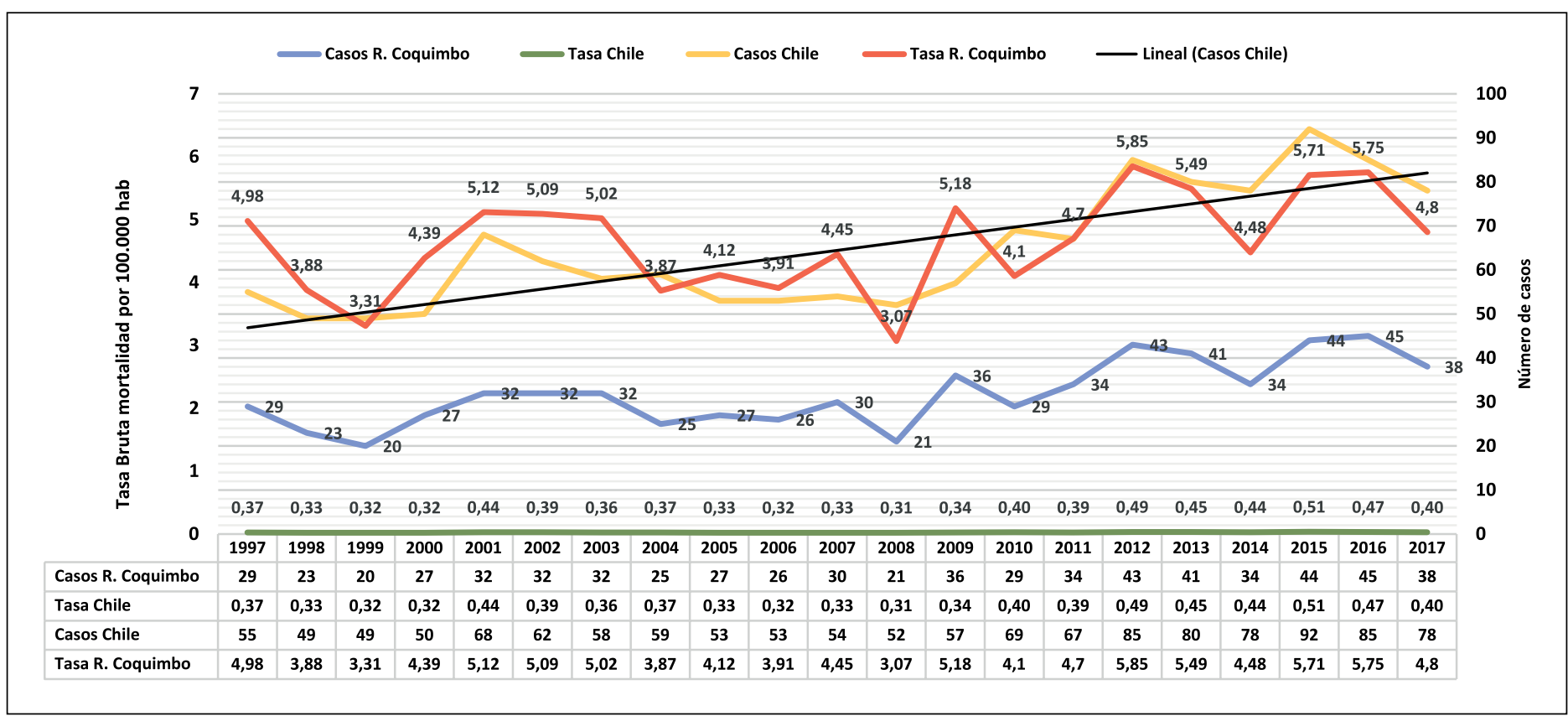

Figura 1. Tendencia de mortalidad por enfermedad de Chagas: casos y tasa bruta por 100.000 habitantes, Chile y Región de Coquimbo.

Figura 2. Variabilidad porcentual de la mortalidad por enfermedad de Chagas entre la Región de Coquimbo y el total país, período 1997 al 2017.

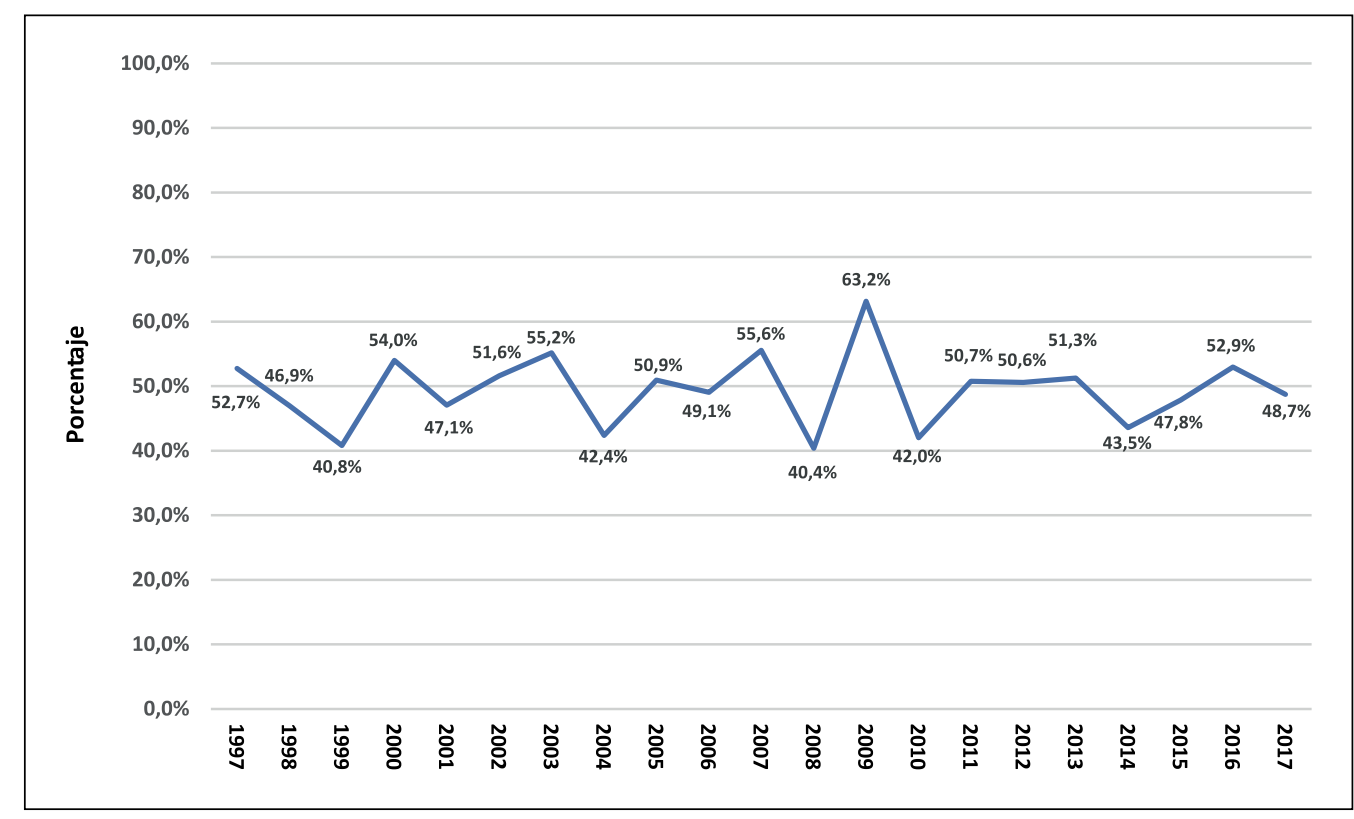

confirmados presentó un número mayor de casos el año 2011 (n: 1.472 casos), de los cuales 991 casos $(67 \%)$ fueron notificados por ENO en la Región de Coquimbo. En el año 2012 fueron notificados 45,8\% (462 de 1.007 confirmados), el 2013 fueron $35 \%$ (139 de 395 confirmados) y en el año 2014, 66\% (530 de 797 confirmados).
Las características demográficas de los casos nuevos confirmados con enfermedad de Chagas presentan diferencias significativas de edad al momento del diagnóstico, donde el promedio de edad de mujeres es de 50 años, versus seis años más tarde (56 años) en los hombres ( $\mathrm{T}$ test: 29,19 valor p: 0,000$)$. Un $12,7 \%$ (375 casos) de los 
casos tienen menos de 20 años al momento del diagnóstico con enfermedad de Chagas en la Región de Coquimbo, y de ellos, 74\% corresponde a niños bajo un año de edad ( 278 casos). La distribución por edad de los casos nuevos diagnosticados entre los años 2011 y 2017 señala que al menos 69,9\% (2.058 casos) tendría menos de 60 años, cumpliendo con uno de los criterios de inclusión para el tratamiento antiparasitario dispuesto en la norma del MINSAL, pero serían excluidos sólo por tener más de 60 años 30,1\% (888 casos con registros de edad en los confirmados por el ISP, como ya fue mencionado, se desconoce la edad en 53\% de los confirmados). Cabe destacar que la distribución por sexo de los menores de 20 años es similar a través de los años, excepto en el último año, donde predominó el diagnóstico en el sexo masculino. En los mayores de 21 años, en cambio, existe una marcada predominancia del sexo femenino en el diagnóstico por enfermedad de Chagas, especialmente en los años 2011 y 2017 (Figura 6).

En la Figura 7 se muestra la distribución espacial a nivel de las 15 comunas en los dos períodos analizados, mostrando una heterogeneidad en las tasas para ambos indicadores. En el caso de las tasas de incidencia acumulada, existen comunas con mayores tasas que son; Ovalle, Monte Patria, Andacollo e Illapel. En cambio, en las tasas de mortalidad acumuladas en 20 años (1997 al 2017), las comunas que presentan mayores tasas fueron; Salamanca, Illapel, Combarbalá y Río Hurtado. Siendo todas ellas, las áreas geográficas más interesantes de estudiar con modelos explicativos e intervenciones futuras (Figura 7).

\section{Discusión}

El alto porcentaje de muertes por enfermedad de Chagas encontrado en la presente investigación en la Región de Coquimbo comparado con los casos totales del país (casi la mitad de los casos fallecidos en todo el territorio residen en esta región), plantea interrogantes importantes de desarrollar en un futuro próximo. Una posible explicación podría deberse a un mayor riesgo de exposición al insecto vector, ya sea el vector doméstico (T. infestans), y/o un vector silvestre Mepraia spinolai ${ }^{7,12}$, y a los cambios climáticos que hacen de la Región de Coquimbo un área donde la enfermedad de Chagas debe continuar previniéndose, tanto ambientalmente como en las personas (vigilancia epidemiológica intensificada). Debemos recordar, además, que la enfermedad puede ser transmitida al hombre y a más de 150 especies de animales domésticos y mamíferos silvestres ${ }^{13}$.

Otra posible explicación al aumento de las tasas encontradas en la región es que la enfermedad de Chagas ha sido estudiada y se ha otorgado tratamientos a personas

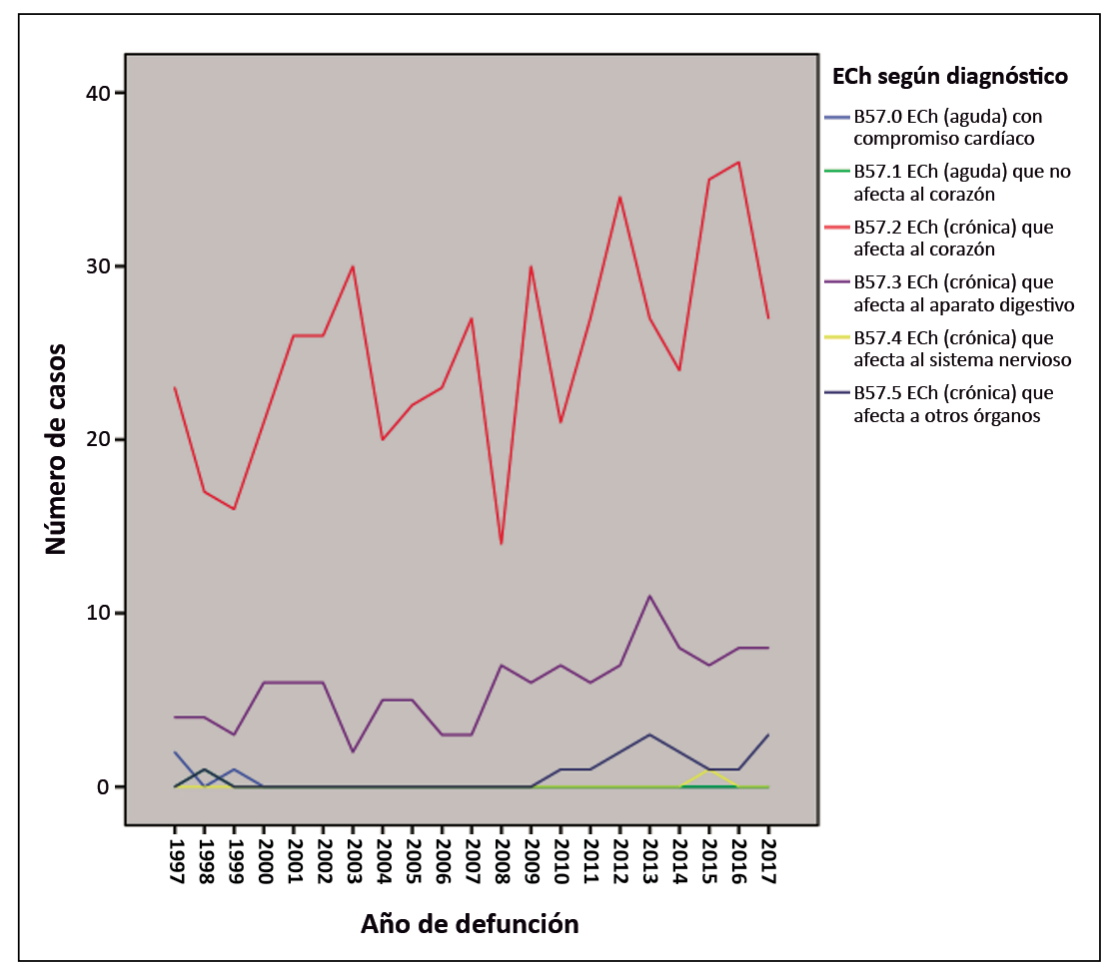

Figura 3. Distribución de casos de mortalidad por enfermedad de Chagas según año y diagnóstico específico, Región de Coquimbo, período 1997 al 2017.

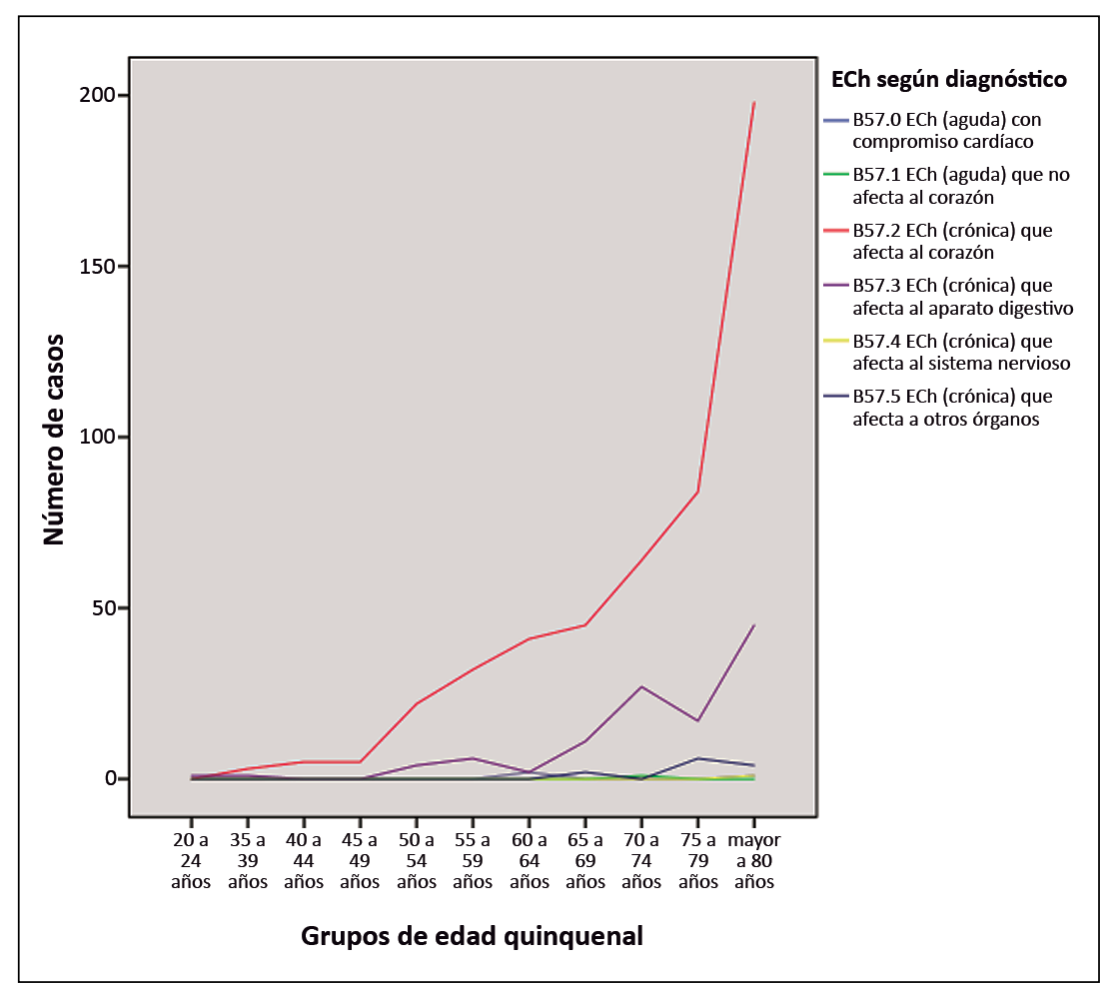

Figura 4. Distribución de los casos de mortalidad por enfermedad de Chagas según grupos quinquenales de edad y diagnóstico específico, Región de Coquimbo, período 1997 al 2017. 
Figura 5. Tasa bruta de incidencia y casos confirmados por el ISP con diagnóstico de enfermedad de Chagas, más casos notificados en la Región de Coquimbo, período 2011 2017.

Figura 6. Casos confirmados por ISP con diagnóstico de enfermedad de Chagas, en menores de 20 años y mayores de 21 años, según sexo y año de diagnóstico, Región Coquimbo, período 2011-2017.
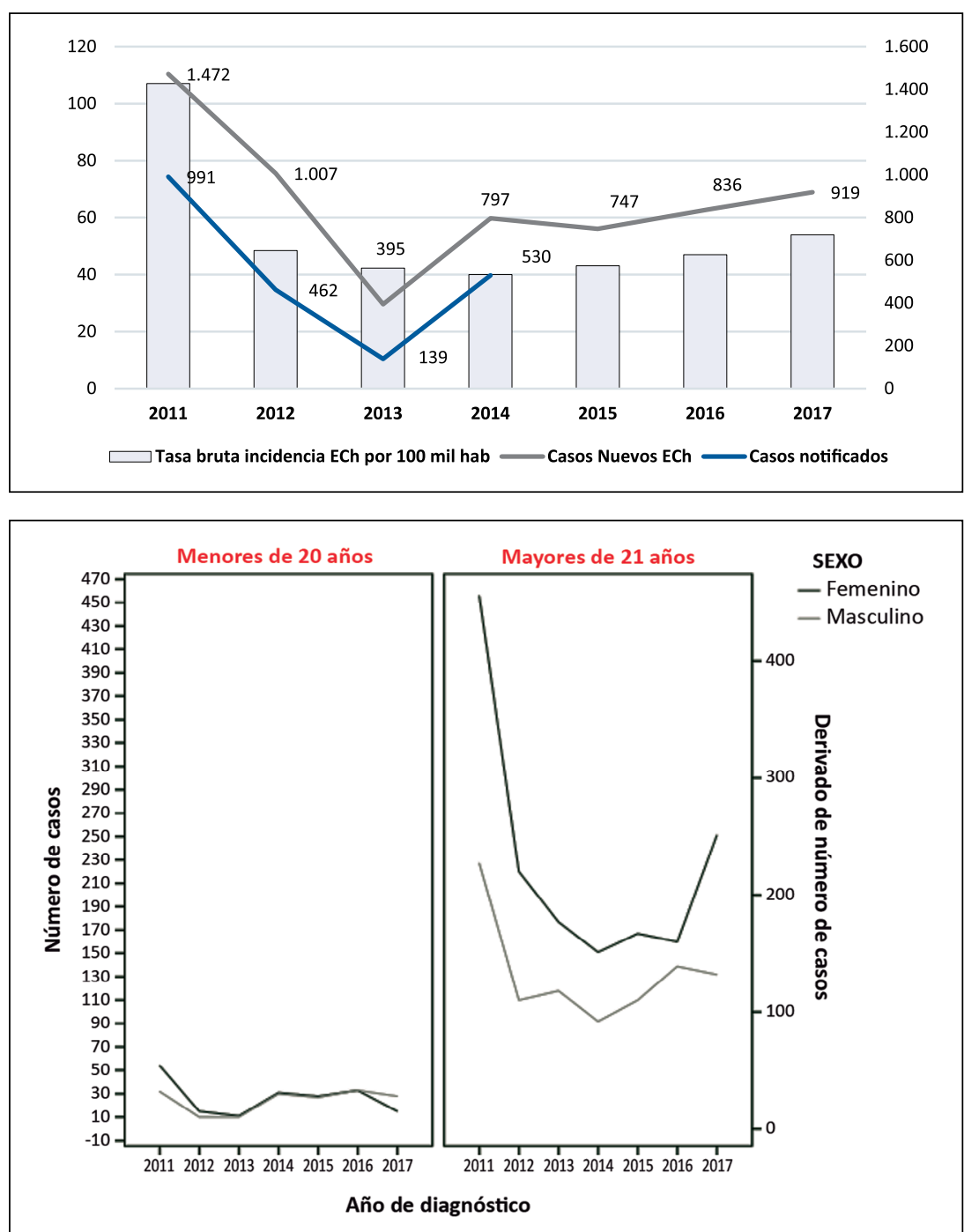

Figura 7. Mapas con distribución geográfica de tasas acumuladas de tasas de incidencia y mortalidad por enfermedad de Chagas en las 15 comunas de la Región de Coquimbo.

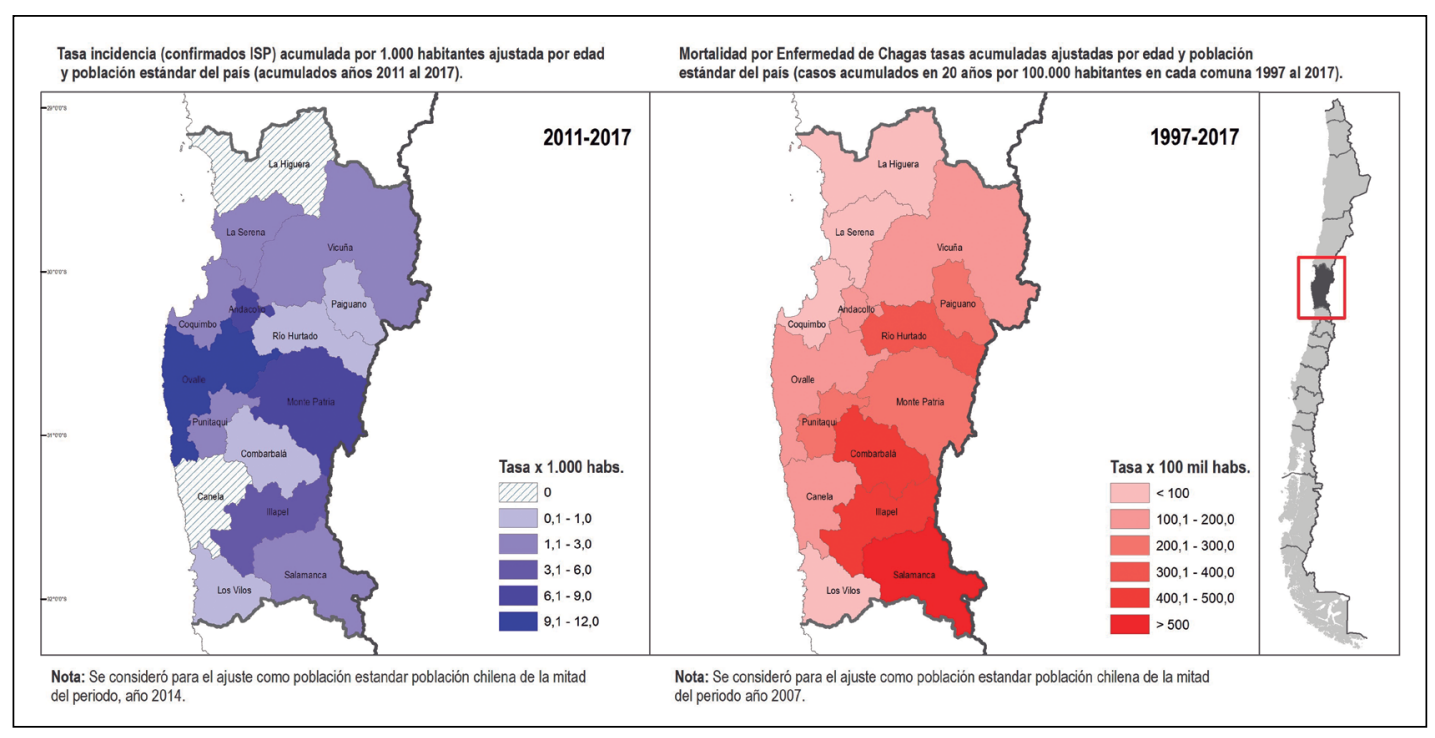


coincide con otras investigaciones, cuando se han realizado cohortes de pacientes con enfermedad de Chagas, que informan constantemente que los hombres tienen un mayor riesgo de enfermedad cardíaca y muerte ${ }^{23,24}$. En nuestro país, por el contrario, son los varones los menos pesquisados y tratados ${ }^{6}$.

Sin embargo, en materias de incidencia las mujeres presentaron el mayor número de casos y tasas incidentes, similar a lo reportado por el informe nacional, donde, además, la diferencia ha ido en aumento entre 2014 y el 2017. Este aumento en el país fue de $71 \%$ más que tasas de notificación del sexo masculino. Se estima que este aumento se debe al mayor acceso a diagnóstico precoz en las mujeres embarazadas y mujeres en edad fértil, no así la búsqueda intencional de casos sospechosos en el caso de los hombres. A pesar de que la notificación es obligatoria, los casos notificados son menos cada año que los reportados por el ISP, y en la Región de Coquimbo, existiría una baja tasa de notificación con respecto al resto del país, según informe de indicadores programáticos para la enfermedad de Chagas 2016 y lo demostrado en esta investigación del análisis de casos confirmados por el ISP y las bases de datos ENO disponibles en el DEÍS-MINSAL ${ }^{6}$. Una posible explicación es la subnotificación, lo que se propone continuar estudiando, especialmente en identificar las causas que la provocan; esto, para favorecer en el futuro la notificación a las personas que deben saber su condición y el derecho de acceso al tratamiento precoz. Por los motivos antes señalados, se decidió el cálculo de las tasas de incidencia de los casos confirmados por el ISP, a pesar de que estas cifras aún no reflejan la verdadera magnitud del problema, pues no contempla a todas las personas infectadas que no se han realizado algún examen para detectar la presencia del parásito en su sangre ${ }^{3,4,6}$.

Respecto a la edad de los casos confirmados, debe ser registrada por los laboratorios que analizan muestras, especialmente con técnicas confirmatorias, para tener una mejor visualización futura de la distribución según grupos de riesgo, en especial los menores de 20 años y los mayores de 60 años. Los casos nuevos o incidentes confirmados por el ISP que tenían incorporada esta variable correspondieron sólo a 46,9\% de la muestra total. A pesar de esta limitante, se logró determinar que los menores de 20 años en la Región de Coquimbo alcanzarían a 375 casos en siete años. Cabe preguntase, ¿estos niños y jóvenes han sido enfermados por sus madres en su totalidad, vía transmisión transplacentaria?, ya que Chile ha certificado la interrupción de la transmisión vectorial de la infección de la enfermedad desde el año 1999 y fue re-certificada el año 2016 por una comisión de expertos de INCOSURChagas y OPS. La interrupción de la transmisión vectorial se hizo bajo parámetros como registro de sólo $0,2 \%$ de infestación domiciliaria por T. infestans, baja densidad 
de ejemplares por vivienda, bajo índice de infección natural por $T$. cruzi en el vector ${ }^{6}$. Se plantea la necesidad de identificar a estos niños y jóvenes, hacer seguimiento y asegurar el acceso a tratamiento precoz a este grupo, pensando en futuras intervenciones en la región debido a la mayor efectividad de la terapia en los menores de edad.

En la actualidad existen modelos matemáticos que tratan de explicar el resurgimiento de la enfermedad de Chagas en zonas infectadas con la presencia de hospederos de reservorio como las ratas, por la propagación geográfica de la infección ${ }^{25}$. Se debe plantear en el actual programa de enfermedad de Chagas, la necesidad de hacer estudio serológico de la familia antecesora (especialmente la línea materna) a estos casos de pacientes bajo 20 años de edad o de familias expuestas, además de seguimiento, acceso al tratamiento, estudios epidemiológicos del vector en otros lugares (no los habituales o históricos), por el posible resurgimiento del vector o presencia de hospederos de reservorio con propagación geográfica de la infección, para asegurarnos que la vía de transmisión predominante en Chile es sólo la vía transplacentaria.

A nivel mundial y producto de las migraciones, la enfermedad de Chagas ha sido planteada como un problema global, dado que existen también casos en lugares no endémicos como Canadá, E.U.A, Europa, Australia y Japón. Por estos motivos, se recomienda, además, un abordaje multidisciplinario a fin de lograr mejores estrategias de control, acceso precoz al diagnóstico y medicamentos, e investigación y tratamiento de las co-morbilidades asociadas con la enfermedad de Chagas crónica ${ }^{26}$.

Existen países como Argentina donde se ha avanzado en legislar, por ejemplo, lo que significa ser portador de la enfermedad de Chagas. La legislación establece el proceder ante serología reactiva para ser considerado como restrictivo para el ingreso a un trabajo, "siempre que no existan otros elementos diagnósticos, clínicos, radiológicos y electrocardiográficos que indiquen la disminución de la capacidad laboral imputable a la infección chagásica". Sin embargo, esto ya está siendo cuestionado como discriminatorio ${ }^{27}$, incluso la Ley 26.281 de Argentina (2007), asignó carácter prioritario a ..."la prevención y control de todas las formas de transmisión de la enfermedad de Chagas, hasta su definitiva erradicación de todo el territorio nacional... "y en su artículo $5^{\circ}$ habla de prohibir "realizar reacciones serológicas para determinar la infección chagásica a los aspirantes a cualquier tipo de empleo o actividad". Se suma la Ley N. 23.592 sobre el tratamiento antiparasitario específico, evitando toda acción dilatoria y la Ley 26.279 que norma la detección y posterior tratamiento de determinadas patologías en el recién nacido donde incluye la enfermedad de Chagas, sancionada en agosto de 2007; finalmente, la Ley 26.945, donde se declara el día nacional por una Argentina sin Chagas, sancionada en julio de 2014.
Se debe avanzar en el acceso al diagnóstico precoz y tratamiento en Chile; esto, a pesar de presentar algunos efectos adversos a los medicamentos, en particular en adultos, ya que los niños lo toleran de mejor manera. Los reportados por investigaciones nacionales serían: náuseas, vómitos, malestar gástrico, anorexia, alteraciones hematológicas, dermatitis, eritema, rash, sensibilidad a la luz, insomnio alucinaciones, otros. A pesar de estos efectos, se ha recomendado que la enfermedad de Chagas debe ser tratada siempre, en su período agudo, período crónico determinado inicial e intermedio y en el período crónico indeterminado ${ }^{28}$. Existe consenso en que la enfermedad de Chagas debe ser tratada en cualquiera de sus períodos, exceptuando los casos crónicos terminales ${ }^{29}$.

Los resultados sobre las personas descritos en la presente investigación son coincidentes con los estudios en el medio ambiente, en detectar como zona de riesgo a la Región de Coquimbo, debido a que el cambio climático parece jugar un papel importante en el resurgimiento de la enfermedad y $T$. infestans. Estudios ambientales señalan que la distribución de $T$. infestans y de la enfermedad de Chagas se correlacionan con la temperatura máxima y la precipitación durante el mes más seco que, sumado a la distribución potencial reportada para $T$. infestans (basados en encuestas), señalan una mayor probabilidad de presencia hacia el Norte de Chile (principalmente en la Región de Coquimbo), considerando que es una de las zonas de mayor riesgo. Por tales motivos, se recomienda mantener los sistemas de vigilancia y control vectorial, e incluso mejorarse, para evitar focos silvestres, intromisión y re-colonización de viviendas humanas. Lo anterior se apoya en que la infestación domiciliaria ha disminuido, pero han aumentado los reportes de intromisión de individuos solitarios y los focos silvestres de $T$. infestans y los índices tripano-triatominos se mantienen con valores altos en todas las especies de vectores ${ }^{7-9}$. Preocupa los bajos sistemas de pesquisa en la población de riesgo y población general en diagnósticos y tratamiento antiparasitario precoz en zonas endémicas.

La evidencia sugiere que el nivel socio-económico también influye en el pronóstico de la complicación cardiovascular consecuente a padecer enfermedad de Chagas. Pero existe contradicción que la enfermedad de Chagas es predominante en las poblaciones pobres; actualmente se describe que además sería un predictor de mortalidad por todas las causas, independientemente del estado socio-económico ${ }^{29}$. A esto se debe sumar que existen escasos estudios o investigaciones en el mundo, y particularmente en Chile, acerca de la calidad de vida, el seguimiento de pacientes con enfermedad de Chagas, especialmente en la etapa asintomática de la enfermedad o en el otro extremo en la etapa sintomática crónica, como es vivir con una patología cardiovascular, digestiva o de otro tipo ${ }^{30}$. 
de vacunas. Una vacuna no solo podría disminuir la carga de infección, sino también la enfermedad cardíaca grave ${ }^{31}$.

Agradecimientos: A Salvador Ayala, del Sub-departamento de Vigilancia de Laboratorio del Instituto de Salud Pública de Chile en la construcción de los mapas, a Werner Apt del Programa de Parasitología, Facultad de Medicina, Universidad de Chile, por su entrega al estudio de esta patología en la población de la Región de Coquimbo y a Mauricio Canals Lambarri, investigador del Departamento de Medicina, Facultad de Medicina, de la Universidad de Chile, por su generosa entrega de conocimiento sobre los factores medio ambientales y del vector.

\section{Referencias bibliográficas}

1.- WHO/Department of control of neglected tropical diseases. Integrating neglected tropical diseases in global health and Development. Fourth WHO report on neglected tropical diseases, 2017, ISBN: 978924156544 8, p.267. https://apps.who.int/iris/bitstream/ha ndle/10665/255011/9789241565448-eng. pdf;sequence $=1$

2.- Encuesta Nacional de Salud - Chile 20162017, [Internet]. Ministerio de Salud-Gobierno de Chile. Disponible en: http://epi.minsal.cl/ resultados-encuestas/ [acceso el 28 de enero de 2020].

3.- Norma general técnica $\mathrm{N}^{\circ} 162$ del Control $\mathrm{y}$ Prevención Nacional de La Enfermedad de Chagas aprobada por resolución exenta $\mathrm{N}^{\circ}$ 144, 2014. Ministerio de Salud-Gobierno de Chile. Disponible en: https://www.minsal.cl/ sites/default/files/NORMA\%20TECNICA CHAGAS_FINAL.pdf

4.- Manual de procedimiento para la atención de pacientes con enfermedad de Chagas. 2017. Ministerio de Salud-Gobierno de Chile. Disponible en: https://diprece. minsal.cl/wrdprss_minsal/wp-content/ uploads/2018/01/2018.01.18_ENFERMEDADDE-CHAGAS-2017.pdf

5.- Fuentes R, Maturana M, De la Cruz R. Eficacia de nifurtimox para el tratamiento de pacientes con enfermedad de Chagas crónica. Rev Chilena Infectol 2012; 29 (1): 82-86. doi. org/10.4067/S0716-10182012000100013.

6.- Informe Estrategia Integrada de Prevención y Control de la Enfermedad de Chagas 2016. Ministerio de Salud-Gobierno de Chile. Disponible: https://diprece.minsal.cl/ wp-content/uploads/2018/10/FOLLETO_ PROGRAMA-CHAGAS.pdf

7.- Tapia-Garay V, Figueroa D, Maldonado A, Frías-Laserre D, Gonzalez Ch, Parra A, et al. Assessing the risk zones of Chagas' disease in Chile, in a world marked by global climatic change. Mem Inst Oswaldo Cruz 2018; 113 (1): 24-9. doi: 10.1590 / 0074 02760170172 .

8.- Hernández J, Núñez I, Bacigalupo A, Cattan PE. Modeling the spatial distribution of Chagas disease vectors using environmental variables and people's knowledge. Int J Health Geogr 2013; 12: 29. doi: 10.1186 / 1476-072X12-29.

9.- Canals M, González Ch, Canals L, Canals A, Cáceres D, Alvarado S, et al. ¿Qué dicen los números de la evolución temporal de la enfermedad de Chagas? Rev Chilena Infectol 2017; 34 (2): 120-7. Disponible en: http:// dx.doi.org/10.4067/S0716-10182017000200004 [citado el 28 de enero de 2020].

10.- Departamento de Estadísticas e Informática de Salud (DEIS), Ministerio de Salud-Gobierno de Chile. [Internet], http://www.deis.minsal.cl, (Consultado en noviembre de 2019).

11.- Instituto Nacional de Estadísticas, Ministerio de Economía Chile: Estimaciones y proyecciones de población por sexo y edad. Total, país: 19502050. Disponible en: https://regiones.ine.cl/ coquimbo/inicio.

12.- Reyes R, Yohannessen K, Ayala S, Canals M. Estimaciones de la distribución espacial del riesgo relativo de mortalidad por las principales zoonosis en Chile: enfermedad de Chagas, hidatidosis, síndrome cardiopulmonar por hantavirus y leptospirosis. Rev Chilena Infectol 2019; 36 (5): 599-606. Disponible en: http:// dx.doi.org/10.4067/S0716-10182019000500599 [citado el 29 de enero de 2020].

13.- Canals M, Ehrenfeld M, Cattan A. Situación de Mepraia spinolai, vector silvestre de la enfermedad de Chagas en Chile, en relación con otros vectores desde la perspectiva de sus fuentes de alimentación. Rev Med Chile 2000; 128(10): 1108-12. Disponible en: http://dx.doi.
org/10.4067/S0034-98872000001000005 [citado el 29 de enero de 2020].

14.- Apt W, Arribada A, Zulantay I, Saavedra M, Araya E, Solari A, et al. Trypanosoma cruzi burden, genotypes, and clinical evaluation of Chilean patients with chronic Chagas cardiopathy. Parasitol Res 2015; 114: 3007-18. https://doi.org/10.1007/s00436-015-4503-2.

15.- Zulantay I, Apt W, Ramos D, Godoy L, Valencia C, Molina M, et al. La relevancia epidemiológica del estudio familiar en la enfermedad de Chagas. PLoS Negl Trop Dis 2013; 7 (2): e1959. https://doi.org/10.1371/ journal.pntd.0001959.

16.- Saavedra M, Zulantay I, Apt W, Martínez G, Rojas A, Rodríguez J. Chronic Chagas disease: PCR-xenodiagnosis without previous microscopic observation is a useful tool to detect viable Trypanosoma cruzi. Biol Res 2013; 46(3): 295-8. http://dx.doi.org/10.4067/ S0716-97602013000300011 [citado el 12 de julio de 2020].

17.- Bustamante J M, Rivarola H W, Fernández A R, Enders J E, Fretes R, Palma J A, et al. Trypanosoma cruzi reinfections in mice determine the severity of cardiac damage. Int J Parasitol 2002; 32: 889-96. doi: 10.1016/ s0020-7519(02)00023-1.

18.- de Oliveira R B, Troncon L E, Dantas R O, Menghelli U G. Gastrointestinal manifestations of Chagas' disease. Am J Gastroenterol 1998; 93: 884-9. https://doi.org/10.1016/S00029270(98)00151-8.

19.- Vicco M H, Rodeles L, Yódice A, Marcipar I. Chagas disease, a risk factor for high blood pressure. Blood Press 2014; 23 (6): 345-8. doi: 10.3109/08037051.2014.907977

20.- Cucunubá Z M, Okuwoga O, Basáñez M G, Nouvellet $P$. Increased mortality attributed to Chagas disease: a systematic review and meta-analysis. Parasit Vectors 2016; 9: 42. doi: 10.1186/s13071-016-1315-x. 
21.- Lee B Y, Bacon K M, Bottazzi M E, Hotez P J. Global economic burden of Chagas disease: a computational simulation model. Lancet Infect Dis. 2013 ; 13(4): 342-8. doi: 10.1016/S14733099(13)70002-1.

22.- Wilson L S, Strosberg A M, Barrio K. Costeffectiveness of Chagas disease interventions in Latin America and the Caribbean: Markov models. Am J Trop Med Hyg 2005; 73: 901-10. PMID: 16282301.

23.- Sabino E C, Ribeiro A L, Salemi VMC, Di Lorenzo-Oliveira C, Antunes A P, Menezes $\mathrm{M}$ M, et al. Ten-year incidence of Chagas cardiomyopathy among asymptomatic Trypanosoma cruzi-seropositive former blood donors. Circulation. 2013; 127: 1105-15. doi: 10.1161 / CIRCULATIONAHA.112.123612.

24.- Basquiera A L, Sembaj A, Aguerri A M, Omelianiuk M, Guzmán S, Moreno-Barral $\mathrm{J}$, et al. Risk progression to chronic Chagas cardiomyopathy: influence of male sex and of parasitaemia detected by polymerase chain reaction. Heart 2003; 89: 1186-90. doi: 10.1136/heart.89.10.1186.

25.- Mahdjoub T, Kribs Ch. Evaluación de la velocidad de invasión de poblaciones de vectores triatominos de la enfermedad de Chagas. Rev Mate Teor Aplic 2020; 27 (1): 73-92. doi: https://doi.org/10.15517/rmta. v27il.39949.

26.- Lidani K C F, Andrade F A, Bavia L, Damasceno F S, Beltrame M H, MessiasReason I J, et al. Chagas Disease: from discovery to a worldwide health problem. Front Public Health 2019; 7: 166. doi: 10.3389/ fpubh.2019.00166. PMID: 31312626; PMCID: PMC6614205.

27.- Storino R, Auger S, San Martino M, Urrutia M I, Jörg M. Aspectos biológicos, psicológicos y sociales de la discriminación del paciente chagásico en Argentina. Rev. Salud Pública 2002; 4 (3): 258-69. Disponible: https:// revistas.unal.edu.co/index.php/revsaludpublica/ article/view/18532/19443.
28.- Apt W, Zulantay I. Estado actual en el tratamiento de la enfermedad de Chagas. Rev Med Chile 2011; 139 (2): 247-57. Disponible en: http://dx.doi.org/10.4067/ S0034-98872011000200016 [citado el 23 de septiembre de 2014].

29.- Apt W. Tratamiento de la enfermedad de Chagas. Parasitol. día. 2020; 23 (3-4): 100-12. http://dx.doi.org/10.4067/S071607201999000300007 [citado el 29 de enero de 2020].

30.- de Campos C B, Yamada A T, Araújo F, Pereira-Barreto A C, Mansur A J. Factores socioeconómicos en el pronóstico de la insuficiencia cardíaca en una cohorte brasileña. Int J Cardiol. 2006; 113: 181-7. doi: 10.1016 / j.ijcard.2005.11.009.

31.- Lee B Y, Bacon K M, Connor D L, Willig A M, Bailey R R. The potential economic value of a Trypanosoma cruzi (Chagas disease) vaccine in Latin America. PLoS Negl Trop Dis. 2010; 4 (12): e916. doi: 10.1371/journal.pntd.0000916. 